\title{
Secure Resource Management and Efficient Mining in Cloud Computing
}

\author{
S.Ramamoorthy \\ Assistant Professor, \\ Department of Computer Science and Engineeing \\ SCSVMV University, Enathur, \\ Kanchipuram,Tamilnadu
}

\author{
S.Rajalakshmi, PhD. \\ Professor \& HOD \\ Department of Computer Science and Engineeing \\ SCSVMV University, Enathur, \\ Kanchipuram,Tamilnadu
}

\begin{abstract}
Cloud computing provide a large scale computing resources to various customers and developers. The resource transformation between customers and cloud maintenance can be easily threatened by the various cyber attacks, because cloud computing provide the service to many customers who are not proven to be trustworthy. Therefore cloud computing system needs to provide some fair and secure resource exchanges between customers. Hence a proposed frequent pattern matching resource protocol allows the cloud service provider to identify the duplication and exchange the resource to an agreed service over the internet in a fair way. Based on signature authentication and Frequent record set matching with the frequently used resource identification technique provide an efficient resource management system in Cloud Computing
\end{abstract}

Keywords: cloud computing, signature authentication, hash function, frequent pattern record set matching; data storage, security

\section{INTRODUCTION}

Cloud computing, a new internet-based technology, has been widely envisioned as the most promising technology of IT enterprise. It manages and schedules the computing resources through network, and constitutes a large computing resources pool which can provide service to users on their demand. The network is called "cloud". Resources in cloud it seems that can be extended unlimitedly, got anytime, used on-demand and paid according to apply. This feature is often called using IT service as water or electricity. It is a distributed processing, parallel processing and Grid Computing development. Together with this new technology, lots of business models which can be described of "X as a service (Xaas)" where X could be infrastructure, platform, software etc [1]. The most representative commercial cloud platforms are Amazon Elastic Compute Cloud (EC2) and Amazon Simple Storage System [2], Google App Engine [3], and Microsoft Azure [4]. All of these service providers have achieved great success in business. The increasing network bandwidth and reliable yet flexible network connections make it even possible that users can now subscribe high quality services from data and software that reside solely on remote data centers. Comparing to building their own infrastructures, all these cloud services are able to save users' Investments significantly by migrating business into the cloud. With the development of cloud computing technologies, a growing number of businesses will be moved into the cloud in the near future [5].

Cloud storage is built on the network computing environment. There are many benefits to move data into the cloud. For example, users do not have to care about the complexities of direct hardware management. But since users store their data in the cloud, it means that they will lose the control of them and more and more worries will come out about the data security. Data security is always an important aspect of quality of service and it is also a key issue in cloud computing. Due to traditional cryptographic primitives for the purpose of data security, protection cannot be directly adopted in the environment of cloud [6]. The data stored in cloud is facing different and neoteric challenges. Recently, the importance of ensuring the data security in cloud computing has been highlighted by researchers and enterprises. A representative solution for ensuring data safety is utilizing a signature authentication based protocol to assess the user identification and expose the cloud options provided by the cloud storage services on behalf of the users upon request. In conventional practice, the third party auditor is operated by an institute which is not related to the cloud service provider. But under the circumstances, it may produce wasting of resource and slowness of response. In the mean time, the possibility of the stoppage of the third party auditor may lead a potential instability to the system. It obviously obstructs the development and application of the cloud storage.

In this paper, propose a new efficient signature based authentication protocol for the secure resource management in cloud service provider with record set match based options for cloud data storage. To prove that the given protocol using the session keys and signature authentication will provide the sufficient security and service to the cloud customers in a fair way. Validation function into the cloud service provides architecture and make it trustful and security. This construction drastically reduces the time of response and the bandwidth of occupation during the communication between the third party auditor and the cloud service provider. Obviously, it makes the cloud service provider simplify its operating steps and reduces the constitution's complexity in cloud computing.

The rest of this paper is organized as follows: Section 2 discusses the models and design goals. Section 3 provides the detailed description of the scheme. Section 4 analyzes in the proposed scheme in terms of its security and performance. Section 5 gives the final conclusion of this paper. 


\section{PROBLEMSTATEMENTS}

\subsection{System modules}

The representative traditional network architecture for cloud data storage is shown in Figure 1.

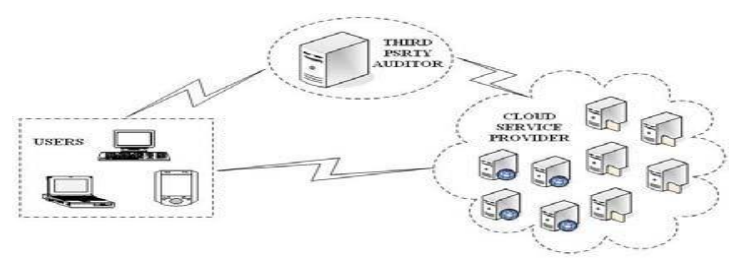

\section{Figure 1 Representative traditional architecture}

It consists of three different network entities which are users, cloud service provider and third party auditor [7]. Users are active participants. They have data to be stored in the cloud and rely on the cloud for data maintenance and computation. Both individual consumers and organizations can be the users. Cloud service provider has significant storage space and computation resource to maintain the users' data. It also has expertise in building and managing distributed cloud storage servers and the ability to own and operate live cloud computing systems. Third party auditor has expertise and capabilities that users do not have and it is trusted to assess and expose risk of cloud storage services on behalf of the users upon request. Similar to [7], users who put their large data files in the cloud storage servers can relieve the burden of storage and computation. At the same time, it is critically important for users to ensure that their data are being stored correctly and security. Users should be equipped with certain security means so that they can make sure their data is safe.

Cloud service provider is always online and assumed to have abundant storage capacity and computation power. The third party auditor is invariably online, too. It makes every data access be in control. In this paper, proposed architecture for cloud storage which is just composed of two parts: Users and Advanced Cloud Service Provider. It is illustrated in Figure 2

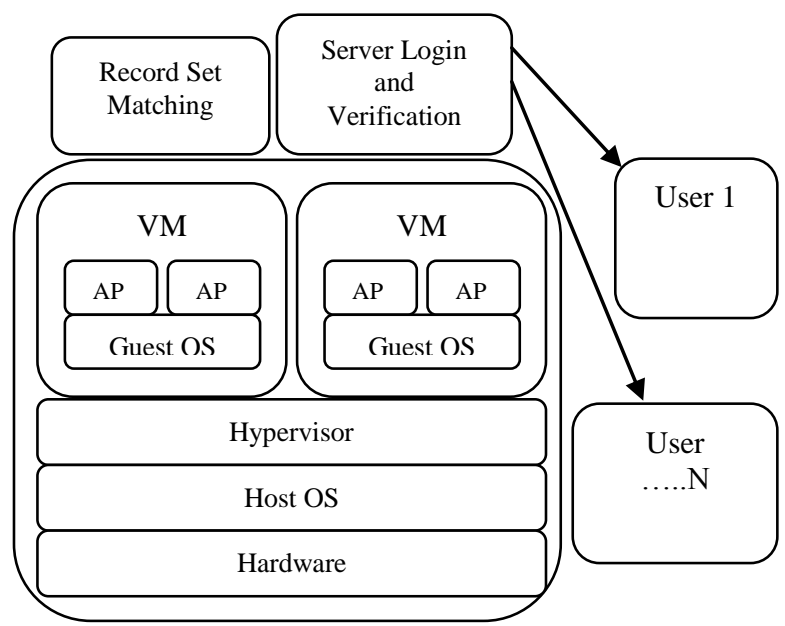

Figure 2 proposed architecture
All the users, and server login and verification of the cloud service provider shown on the figure have the same function discussed above. The main difference between proposed architecture and the traditional one is that the server verification and signature authentication and record set options are different.

\subsection{Security modules}

In this work, client assume that the point-to-point communication channels between users and advanced cloud service provider is encrypted reliably and able to resist all kinds of attacks from adversary. Data is indeed stored appropriately and kept intact all the time in the cloud storage servers. Unauthorized users login, attacks from the different intruders and appending their data files in the cloud have correctness assurance. Data is available against Byzantine failures, malicious data modification and server colluding attacks [8]. Users would try to access data either within or outside the scope of their access privilege. The cloud service provider is more interested in user access privilege, public/private key and other cloud service options information are validated in a secret way.

\subsection{Design goals}

To ensure the data stored security in cloud, user aim to design a efficient mechanism for user identification function into the cloud service provider and achieve the following goals: (1) Access control safety: to ensure both users and cloud service provider must communicate with each other with a privilege given by a certain authentication module.(2)Signature Authentication of the given user provide data trustful in cloud storage servers: to forbid the users and cloud service provider to acquire any authentication information which they are not wanted to.(3)Authentication information efficiently in use: to exchange and make backups authentication information validly in cloud service provider, design a reasonable method to make cloud service provider interior can operate efficiently. (4)Reduced searching Cost by Record set Match (5) Security through Hash function

\section{SECURE RESOURCE PROTOCOL}

\subsection{Main idea}

The cloud service provider can achieve security, trustful and independent, and uniquely utilize the following advanced cryptographic techniques: RSA Based Digital signature with Hash function. RSA algorithm is used to create Digital signature for the given user and encrypt all the session secret keys for the cloud server login request from the user in the advanced cloud service provider. RSA is one of the first great advances public key cryptography. It is believed to be sufficiently secure given long keys and the use of up-to-date implementations. So it can efficiently protect data flow in the proposed scheme. A Record set match based technique from the data mining is used to identify the frequently used resource and user to provides the service options related with the user query from the cloud server

It allows two parties that have no prior knowledge of each other to jointly establish a shared secret key over an insecure communications channel. Also add a Certificate header in front of each file data packet. With this Certificate header, and signature users and cloud service provider can 
communicate with each other safely and directly without a third party auditor server. Each cloud storage servers have the ability to add, identify and update this information header for users.

\subsection{Definition and notation}

Each user is allocated a session key and the own Signature

\subsubsection{Proposed Scheme:}

\section{User Request for the service:}

Register with the given protocol by its own signature Get session key, Certificate, Login, pwd for the Particular session.

Server side operation: Authenticate the given user based on the digital signature. Valid user is only allowed to log in to the cloud service. Otherwise denial the access entry.

Record set Match: User Login into the server the Record set match finds the frequently used available service and options in a feasible cost. User has to select required service and pay for that service. User uses the resource. Session Terminate.

The cloud service provider can achieve security, trustful and independent; and uniquely utilize the following advanced cryptographic techniques: RSA Based Digital signature with Hash function. RSA algorithm use to create Digital signature for the given user and encrypt all the session secret keys for the cloud server login request from the user in the advanced cloud service provider. RSA is one of the first great advances public key cryptography. It is believed to be sufficiently secure given long keys and the use of up-todate implementations. So it can efficiently protect the data flow in the proposed scheme. A Record set match based technique from the data mining is used to identify the frequently used resource and user to provides the service options related with the user query from the cloud server.

With this information header, users and cloud service provider can communicate with each other safely and directly without a third party auditor server. Each cloud storage servers have the ability to add, identify and update this message header for users.

\subsection{Definition and notation}

Each user is allocated a session key and the own signature which are necessary for the cloud access control. Bilinear Diffie-Hellman protocol is used to exchange these keys and signatures between users and cloud service provider. For each data file, users add a message header before sending it to cloud. RSA is used to encrypt the data packet with the allocated keys. In cloud service provider, there are a very small number of servers which are responsible for keeping the whole access keys called trustful organization's servers. They are maintained by a trustful organization and cloud service provider and users cannot get any authentication information from them without a certain module. All of the cloud storage servers have the certain module. It is used to authenticate the users. Also it has the ability to assign and update keys for the users. Each authentication module can communicate with each other and the trustful organization's servers. Figure 2 gives the description of notation to be used in the scheme

\begin{tabular}{|c|l|l|}
\hline SI.No & Notation & Explanation \\
\hline 1. & CUID & Customer Identity \\
\hline 2. & SID & Server Identity \\
\hline 3. & SK & Session Key Identity \\
\hline 4. & SGID & Signature Identity \\
\hline 5. & H-(x) & Hash Function \\
\hline 6. & PWD & Password \\
\hline
\end{tabular}

Figure 3 gives the description of notation

System Setup Before the cloud service provider comes into service. Each cloud storage server will be allocated a SID uniquely by the trustful organization's server. And each cloud storage server can locate all others by SID in cloud. The servers' location information is updated at normal intervals. To make all cloud storage servers have the ability to distribute and update location for the verification protocol for users. And the trustful organization's Verification protocol take charge to maintain all keys which are stored in all cloud storage servers.

Keys and Other Information Exchange If a Verification protocol creates or updates keys for a user, it will send a copy to current server a trustful organization's server at the first time. Also it reduces the validation timing at the server end. The verification protocol has several back-up for the various users in cloud and trustful organization's server has the ability to make backups either.

. When a verification protocol receives a request from a user to download data file,or other services firstly it will check the type of service required and generate a new session key SID and make a copy of signature identity SGID for that particular user. Protocol send the certificate and current login and password for the user to get the service from cloud server. Then it locates the server by SID and send a packet include the CUID in user's $\mathrm{H}-\mathrm{x}$ to the certain server. The certain server will check its UL and return the response with the SessionKey SK of the user. If the server located by SID is a crash machine, request will be sent to the trustful organization's server. The trustful organization's server will do the same work as introduced above. user depict the procedure of exchanging keys and other information .

\subsection{Scheme description}

For clarity author will present the proposed scheme in two relational parts: 1) users and cloud storage servers;

2) cloud storage servers and trustful organization's servers

Interoperation between the users and the cloud storage servers: The interoperation between the users and the cloud storage servers in the proposed scheme are designed as follows.

System Setup In this operation, user sends a request to any one cloud storage server for the purpose to get the resource service from cloud. A Verification protocol gets the request and creates a pair of SK and PWD and a SID for the user uniquely. Then it sends SK, PWD and SID of itself back to the user. Also it will store the keys in its certain module and add the user's information in its USL. At the same time check the feasible service options and server. 
New Signature Creation Before Login into Server the data files to cloud service provider, the user deals with the data file as follow two steps. 1) Create new self signature with the given session key and make the cryptographic digital signature with hash function . 2)Record set match will provide the feasible options for the user. header is 2)Encrypt the Signature and Session key ( include H-x) with CUID. Then send the generated new Signature. The cloud storage server firstly checks up the $\mathrm{H}-\mathrm{x}$ of the Signature and picks up the SID information. Then it searches the SID both in its SL and the trustful organization's servers. If the SID is not found, the server will discard the request. To the contrary, it will communicate with the SID server with the CUID in order to get the available information to the user.

\section{(2) Interoperation between the cloud storage} servers and the trustful organization's servers:

The interoperation between the cloud storage servers and the trustful organization's servers in scheme are designed as follows. Description of exchanging process It is worth nothing that all these process are done by the authentication modules in the cloud storage servers and it does not have any relationship with other modules.

\section{Algorithm used in the proposed Scheme:}

\section{FP-growth : Frequent Pattern Mining}

Find all frequent item sets Generate strong association rules from the frequent item sets. Apriori algorithm is mining frequent item sets for Boolean associations rules and mining Frequent Patterns Without Candidate Generation ,Grow long patterns from short ones using local frequent items. "abc" is a frequent pattern,Get all cloud transactions having "abc": DB $\mid \mathrm{abc}$, "d" is a local frequent item in $\mathrm{DB} \mid \mathrm{abc} \rightarrow$ abcd is a frequent pattern

\begin{tabular}{lll} 
TID & Items bought & (ordered) frequent items \\
\hline 100 & $\{f, a, c, d, g, i, m, p\}$ & $\{f, c, a, m, p\}$ \\
200 & $\{a, b, c, f, l, m, o\}$ & $\{f, c, a, b, m\}$ \\
300 & $\{b, f, h, j, o, w\}$ & $\{f, b\}$ \\
400 & $\{b, c, k, s, p\}$ & $\{c, b, p\}$ \\
$\mathbf{5 0 0}$ & $\{a, f, c, e, l, p, m, n\}$ & $\{f, c, a, m, p\}$
\end{tabular}

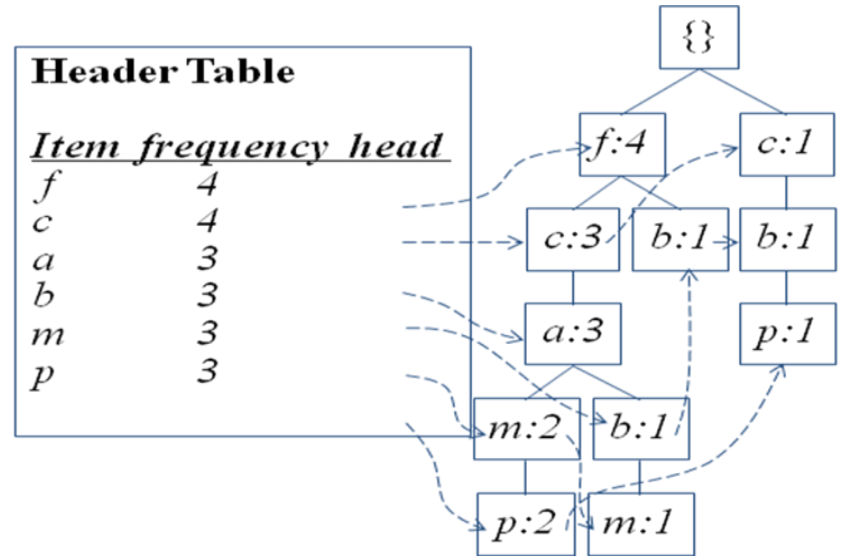

Figure 4 Construct FP-tree from a Transaction Database

\section{F-list=f-c-a-b-m-p}

Scan cloud Database once, find frequent 1-itemset (single item pattern),Sort frequent items in frequency descending order, $\mathrm{f}$ list,Scan DB again, construct FP-tree mining Frequent Patterns With FP-trees using Frequent pattern growth,Recursively grow frequent patterns by pattern and database partition

\section{Evaluation and Results:}

In this cloud frequent pattern mining based resource transformation can be performed based on the following equations

Frequent service access $=$ Repeatedly used service

Total no.of cloud Database

Average

Frequent Item service $=$ Number of Repeated Itemset

$=9 / 10=0.9$ for 9 out of 10 user using the same service

Single Cloud Database

Frequent service $=$ Average number of users

\section{Total number of cloud Database}

Let us consider, most of the cloud users interested for server machine software windows server 2008Then the cloud service provider can offer the winserver2008 for the same set of user request from the client.Using frequent pattern mining cloud server analyze the entire cloud database for the same set of services and recommend to the user for most user frequent service can rapidly increase the number of users in the cloud environment.

\subsection{Result Comparison:}

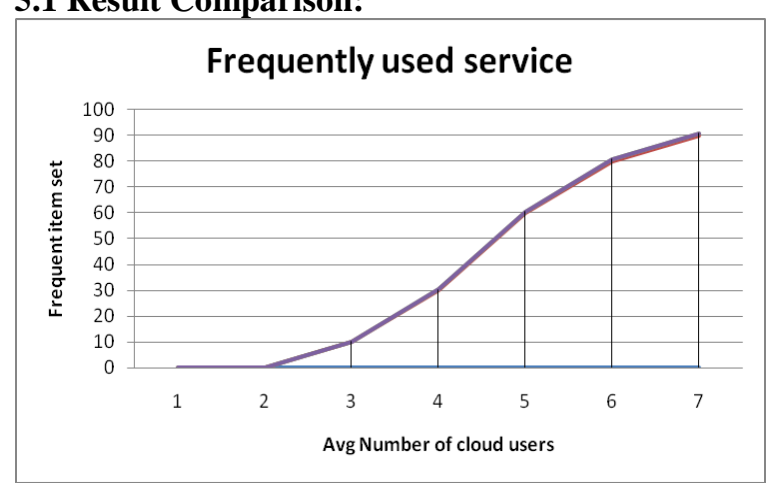

Figure 5 Frequent Service used in cloud Database 


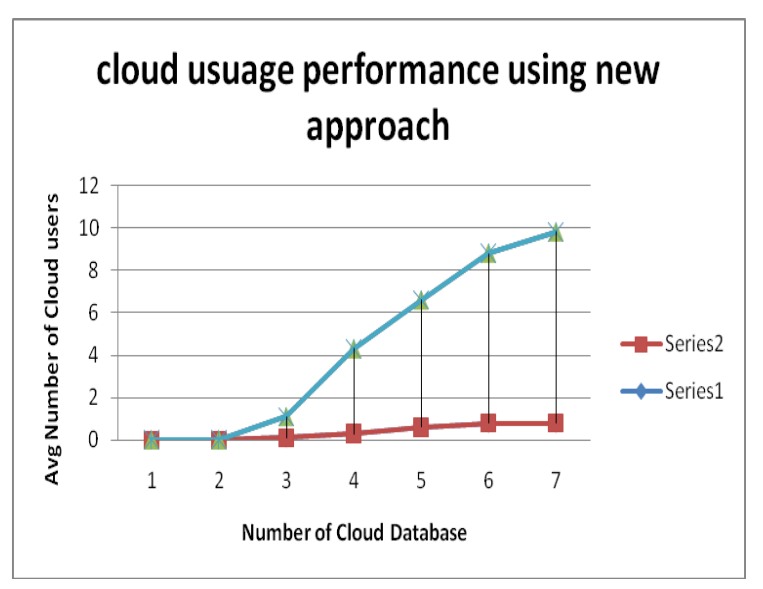

Figure 6 Cloud Performance using new approach

\section{CONCLUSIONS}

This paper aims at designing a Resource management verification protocol with frequently used record set based mining scheme in cloud computing. Investigated the problem of data security in cloud data storage, which is essentially a distributed storage system. To ensure each data access in control and reduce the complexity of cloud computing, In the proposed a scheme using RSA digital signature authentication and record set match based techniques Moreover, the design a self signature authentication mechanism to accomplish the authentication function with the minimum cost. Confidentiality of users'

\section{ACKNOWLEDGEMENTS}

This work was supported by my research guide and different cloud computing international journals.

\section{REFERENCES}

[1] M.Armbrust,A.Fox,R.Griffith,A.D.J oseph,R. H. Katz, A.Konwinski, G. Lee, D.

[2] Amazon Web Services, Online at http://aws.amason.com.

[3] Google App Engine, On line at http://code.google.com/appengine/.

[4] Microsoft Azure,Online.

[5] Shucheng $\mathrm{Yu}$, Cong Wang, Kui Ren, Wenjing Lou, "Achieving Secure, Scalable, and Fine-grained Data Access Control in Cloud Computing", IEEE INFOCOM 2010 proceedings.

[6] M. R. Tribhuwan, V. A. Bhuyar, Shabana Pirzade, "Ensuring Data Storage Security in Cloud Computing through Two-way Handshake based on Token Management", IEEE ARTCom 2010

[7] Qian Wang, Cong Wang, Jin Li, Kui Ren, Wenjing Lou, "Enabling Public Verifiability and Data Dynamics for Storage Security in Cloud Computing”, ESORICS 2009

[8] Cong Wang, Qian Wang, Kui Ren, Wenjing Lou,"Ensuring Data Storage Security in Cloud Co mputing", IEEE, IWQoS. $\quad 17^{\text {th }}$ International 2009.

[9] Lifei Wei, Haojin Zhu, Zhenfu Cao, Weiwei Jia, thanasios V. Vasilakos, "SecCloud: Bridging Secure Storage and Computation in Cloud", IEEEICDCSW,2010 